\title{
Die Berufsstruktur des Deutschen Bundestages - 18. Wahlperiode
}

\section{Melanie Kintz}

Die Bundestagswahl am 22. September 2013 brachte erneut erhebliche personelle Veränderungen im Parlament mit sich. Von den 631 gewählten Abgeordneten zogen 217 erstmalig in den Bundestag ein. Prozentual entspricht dies einem Anteil von 34,6 Prozent und ist primär auf das Ausscheiden der FDP zurückzuführen. Hinzu kam, dass sich circa $100 \mathrm{Ab}-$ geordnete gar nicht wieder zur Wahl gestellt hatten. Dazu gehörten Ilse Aigner (CSU), die in die Landesregierung von Bayern eintrat und der langjährige Bundestags(vize)präsident Wolfgang Thierse (SPD), der in den Ruhestand ging. Des Weiteren verpassten einige Abgeordnete der Grünen und der Linkspartei den Wiedereinzug ins Parlament.

Um bei der folgenden Analyse der Sozialstruktur im 18. Deutschen Bundestag Vergleichbarkeit mit den früheren Wahlperioden zu gewährleisten und Entwicklungslinien darstellen zu können, wird das von Adalbert Hess entwickelte Kategorienschema verwendet. ${ }^{1}$ Nach einer Darstellung der allgemeinen und fraktionsspezifischen Entwicklungen in der Berufsstruktur wird es vor allem um einen Vergleich zwischen ost- und westdeutschen Abgeordneten gehen, um mögliche Angleichungstendenzen herauszuarbeiten. Wie bereits für den 16. und 17. Bundestag wird dabei wieder ein besonderes Augenmerk auf die jüngeren Abgeordneten gerichtet, um einerseits neue Entwicklungstrends in der Berufsstruktur allgemein aufzudecken und andererseits zu untersuchen, inwieweit sich bei der Generation, die ihre Ausbildung größtenteils im wiedervereinigten Deutschland erhalten hat, Karrierewege und Berufsprofile angeglichen haben.

\section{Methodik ${ }^{2}$}

Ausgangsbasis für die Datenerhebung waren erneut die Website des Bundestags, und die Webauftritte der einzelnen Abgeordneten und Fraktionen. Gemäß dem Kategorienschema von Adalbert Hess wurde als Basis für die Zuordnung in Berufsgruppen und Untergruppen der Beruf verwendet, den der Abgeordnete vor Eintritt in den Bundestag innehatte (so genannter Vorberuf). War ein Abgeordneter bereits vor seinem Eintritt in den Bundestag Mitglied eines Parlamentes auf anderer Ebene (Land, Europa), so wurde der Beruf als Grundlage genommen, den er vor Annahme des ersten Mandats ausübte. Auch wenn man nicht eindeutig sagen kann, in welchem zeitlichen Umfang die Abgeordneten vor Bundestagseintritt ihre berufliche Tätigkeit ausübten, so waren doch 87,2 Prozent der Mitglieder des 18. Bundestags vor diesem Mandat berufstätig. Bei den Neulingen sind es gar 91,8 Prozent.

1 Adalbert Hess hat dieses Kategorienschema 1971 entwickelt und verwendete es erstmals in Heft 4/1983 der ZParl zur Dokumentation der Berufsstruktur der Mitglieder des Bundestages. Vgl. ders., Berufsstatistik des 10. Deutschen Bundestages, in: ZParl, 14. Jg. (1983), H. 4, S. 486 - 489.

2 Die Probleme und methodischen Schwierigkeiten bei der Datenanalyse sollen hier nicht weiter erörtert werden. Siehe dazu Franziska Deutsch / Suzanne S. Schüttemeyer, Die Berufsstruktur des Deutschen Bundestages - 14. und 15. Wahlperiode, in: ZParl, 34. Jg. (2003), H. 1, S. 21 - 32 oder Peter Schindler, Datenhandbuch zur Geschichte des Deutschen Bundestages 1949-1999, Bd. 1, Baden-Baden 1999, S. 678 ff. 
Wie bereits in den vorangegangenen Analysen wurden die MdBs, die 1990 Staatsbürger der DDR waren, als „Ostdeutsche“ kategorisiert. Der Geburtsort allein wurde dabei nicht berücksichtigt. Dies hat zur Folge, dass in Ostdeutschland gewählte ${ }^{3}$ westdeutsche Abgeordnete (wie Frank-Walter Steinmeier) als Westdeutsche in die Untersuchung eingehen und Ostdeutsche, die in den alten Bundesländern in den Bundestag gewählt wurden (zum Beispiel Sahra Wagenknecht), als Ostdeutsche. Abgeordnete, die in einem anderen Land geboren wurden und dessen Staatsbürgerschaft besaßen, wurden als „Andere“ kodiert. In der 18. Wahlperiode trifft dies auf zwölf Parlamentarier zu. ${ }^{4}$

Kein Mitglied des 18. Bundestages wurde nach der Wiedervereinigung 1990 geboren; die jüngsten Abgeordneten waren im Wahljahr 26 Jahre alt. Zu den gesondert betrachteten jungen Abgeordneten wurden Personen bis einschließlich 40 Jahre (Geburtsjahr 1973) gezählt. Diese haben bereits mehr als die Hälfte ihres Lebens im wiedervereinigten Deutschland verbracht und waren zum Zeitpunkt des Mauerfalls maximal 16 Jahre alt. Es bleibt daher abzuwarten, ob die Angehörigen dieser Kohorte ähnliche oder doch unterschiedliche Wege in der Berufswahl einschlugen. Außerdem gibt die junge Gruppe einen ersten Hinweis auf zukünftige Entwicklungstendenzen in der Berufsstruktur des Bundestages. ${ }^{5}$

\section{Allgemeine Entwicklungstrends in der Berufsstruktur des Deutschen Bundestages}

Wie bereits erwähnt, wurde ein hoher Anteil neuer Abgeordneter in den Bundestag gewählt. Durch die Einführung der Ausgleichsmandate erweiterte sich der Bundestag von 622 Mandaten in der 17. Wahlperiode auf nunmehr 631. Die FDP, die in der letzten Wahlperiode 93 Abgeordnete stellte, verpasste mit 4,8 Prozent der Wählerstimmen den Wiedereinzug in den Bundestag. Dafür konnten CDU und CSU ihr Ergebnis um 7,7 Prozentpunkte verbessern und damit ihren Sitzanteil stark ausbauen. Die Unionsfraktion besteht jetzt aus 311 Abgeordneten, ein Zuwachs von 72 Mandaten. Auch die SPD, die prozentual ihren Stimmenanteil nur wenig verbessern konnte (um 2,7 Punkte auf 25,7 Prozent) vergrößerte ihre Fraktion von 146 auf 193 Sitze. Die beiden verbleibenden kleinen Fraktionen haben Stimmenanteile und Mandate verloren und sind mit nahezu gleich vielen Abgeordneten im Bundestag vertreten. Die Linkspartei ist drittgrößte Kraft mit 8,6 Prozent der Zweitstimmen und 64 Bundestagsmandaten (minus zwölf Sitze), während die Grünen mit 8,4 Prozent der Zweitstimmen und 63 Abgeordneten (minus fünf Sitze) im Parlament vertreten sind. Trotz der Verluste gibt es auch bei Linken und Grünen neue Mitglieder; letztere verzeichnen 15 Neulinge (23,8 Prozent), in der Fraktion der Linkspartei sind es elf (17,2 Prozent). Der Großteil der neuen Abgeordneten ist allerdings in den beiden Volksparteien zu finden. 106 bei der Union (34,8 Prozent) und 85 in der SPD-Fraktion (44,0 Prozent). ${ }^{6}$

3 Ausgenommen ist Berlin.

4 Diese sind allerdings nicht die einzigen Abgeordneten mit Migrationshintergrund, denn es gibt auch MdBs, die in Deutschland geboren wurden und Eltern beziehungsweise Elternteile mit Migrationshintergrund haben. Dies trifft auf insgesamt 31 Abgeordnete des 18. Bundestages zu.

5 Für frühere WP vgl. die Beiträge von Melanie Kintz in Heft 3/2006 und 3/2010 der ZParl.

6 Für eine vertiefende Analyse der sozio-demographischen Profile und politischen Karrierewege dieser neuen Abgeordneten vgl. Melanie Kintz, Many New Faces, but Nothing New? The Sociodemographic and Career Profiles of German Bundestag Members in the Eighteenth Legislative Period, in: German Politics and Society, 32. Jg. (2014), H. 3, S. $16-25$. 
Tabelle 1: Berufsstatistik der 17. und 18. Wablperiode (Spaltenprozent in Klammern)

\begin{tabular}{|c|c|c|}
\hline & $\begin{array}{l}\text { 17. Bundestag } \\
\text { Stand: } 1 \text {. Januar } \\
2010\end{array}$ & $\begin{array}{l}\text { 18. Bundestag } \\
\text { Stand: } 1 \text {. Februar } \\
2014\end{array}$ \\
\hline Abgeordnete insgesamt & 622 & 631 \\
\hline $\mathrm{CDU} / \mathrm{CSU}$ & 239 & 311 \\
\hline SPD & 146 & 193 \\
\hline FDP & 93 & - \\
\hline Die Linke & 76 & 64 \\
\hline Grüne & 68 & 63 \\
\hline Die Berufsgruppen ${ }^{1}$ & 17. Bundestag & 18. Bundestag \\
\hline \multicolumn{3}{|l|}{ 1. Beamte } \\
\hline a) So genannte politische Beamte & 9 & 9 \\
\hline b) Andere Beamte des höheren Dienstes (Verwaltung) & 39 & 46 \\
\hline $\begin{array}{l}\text { c) Beamte des gehobenen und mittleren Dienstes } \\
\text { (Verwaltung) }\end{array}$ & 14 & 21 \\
\hline d) Richter und Staatsanwälte & 12 & 4 \\
\hline e) Berufssoldaten & 2 & 2 \\
\hline f) Kommunale Wahlbeamte & 30 & 39 \\
\hline g) Professoren an Universitäten und Hochschulen & 9 & 8 \\
\hline $\begin{array}{l}\text { h) Andere Wissenschaftler an Universitäten und } \\
\text { Hochschulen }\end{array}$ & 31 & 24 \\
\hline i) Lehrer an Gymnasien (u.ä.) & 21 & 13 \\
\hline j) Lehrer an Grund-, Hauptschulen und Realschulen (u.ä.) & 17 & 17 \\
\hline Beamte zusammen & $\begin{array}{r}184 \\
(29,6 \%) \\
\end{array}$ & $\begin{array}{r}183 \\
(29,0 \%) \\
\end{array}$ \\
\hline $\begin{array}{l}\text { 2. Angestellte des öffentlichen Dienstes } \\
\text { (auch von Körperschaften, Anstalten und Stiftungen des öf- } \\
\text { fentlichen Rechts) zusammen }\end{array}$ & $\begin{array}{r}31 \\
(5,0 \%)\end{array}$ & $\begin{array}{r}20 \\
(3,2 \%)\end{array}$ \\
\hline a) Bedienstete der EG/EU zusammen & 1 & 1 \\
\hline 3. (Ev.) Pfarrer und Diakone zusammen & $\begin{array}{r}3 \\
(0,5 \%) \\
\end{array}$ & $\begin{array}{r}2 \\
(0,3 \%)\end{array}$ \\
\hline \multicolumn{3}{|l|}{$\begin{array}{l}\text { 4. Angestellte von politischen und gesellschaftlichen Or- } \\
\text { ganisationen }\end{array}$} \\
\hline a) Angestellte von Parteien, Fraktionen (u.ä.) & 58 & 73 \\
\hline $\begin{array}{l}\text { b) Angestellte von Gewerkschaften und Arbeitnehmer- } \\
\text { organisationen }\end{array}$ & 20 & 22 \\
\hline $\begin{array}{l}\text { c) Angestellte sonstiger Organisationen und Institutionen } \\
\text { mit politischen, gesellschaftlichen, kulturellen und } \\
\text { karitativen Zielsetzungen }\end{array}$ & 25 & 29 \\
\hline Angestellte dieser Organisationen zusammen & $\begin{array}{r}103 \\
(16,6 \%) \\
\end{array}$ & $\begin{array}{r}124 \\
(19,7 \%) \\
\end{array}$ \\
\hline $\begin{array}{l}\text { 5. Angestellte der Wirtschaft } \\
\text { (in Industrie, Handel, Handwerk, Gewerbe, und } \\
\text { entsprechenden Verbänden) zusammen }\end{array}$ & $\begin{array}{r}84 \\
(13,5 \%)\end{array}$ & $\begin{array}{r}93 \\
(14,7 \%)\end{array}$ \\
\hline
\end{tabular}




\begin{tabular}{|c|c|c|}
\hline \multicolumn{3}{|l|}{ FortsetzungTabelle 1} \\
\hline \multicolumn{3}{|l|}{$\begin{array}{l}\text { 6. Selbständige } \\
\text { (oft zugleich in entsprechenden Verbänden tätig) }\end{array}$} \\
\hline $\begin{array}{l}\text { a) Selbständige in Industrie, Handel Handwerk und } \\
\text { Gewerbe }\end{array}$ & 48 & 46 \\
\hline b) Selbständige in Land- und Forstwirtschaft & 12 & 15 \\
\hline Selbständige zusammen & $\begin{array}{r}60 \\
(9,7 \%) \\
\end{array}$ & $\begin{array}{r}61 \\
(9,7 \%)\end{array}$ \\
\hline \multicolumn{3}{|l|}{ 7. Angehörige freier Berufe } \\
\hline a) Rechtsanwälte und Notare ${ }^{2}$ & 69 & 58 \\
\hline $\begin{array}{l}\text { b) Angehörige anderer freier Berufe } \\
\text { (Ärzte, Apotheker, Ingenieure, Steuerberater, Schriftstel- } \\
\text { ler, Journalisten u.ä.) }\end{array}$ & 32 & 29 \\
\hline Freiberufler zusammen & $\begin{array}{r}101 \\
(16,3 \%) \\
\end{array}$ & $\begin{array}{r}87 \\
(13,8 \%) \\
\end{array}$ \\
\hline 8. Hausfrauen zusammen & $\begin{array}{r}3 \\
(0,5 \%) \\
\end{array}$ & $\begin{array}{r}4 \\
(0,6 \%) \\
\end{array}$ \\
\hline $\begin{array}{l}\text { 9. Arbeiter zusammen } \\
\text { (ohne solche Abgeordnete, die zwar beruflich als Arbeiter } \\
\text { begonnen haben, jetzt aber anderen Gruppen zuzurechnen } \\
\text { sind) }\end{array}$ & $\begin{array}{r}2 \\
(0,3 \%)\end{array}$ & $\begin{array}{r}1 \\
(0,2 \%)\end{array}$ \\
\hline $\begin{array}{l}\text { 10. Sonstige zusammen } \\
\text { (darunter in Ausbildung befindliche, Arbeitslose oder bisher } \\
\text { ohne Berufsausübung) }\end{array}$ & $\begin{array}{r}27 \\
(4,4 \%)\end{array}$ & $\begin{array}{r}28 \\
(4,4 \%)\end{array}$ \\
\hline $\begin{array}{l}\text { 11. Nicht verwendbare Angaben } \\
\text { (fehlende genaue Angaben im Sinne des vorliegenden } \\
\text { Schemas) }\end{array}$ & $\begin{array}{r}24 \\
(3,9 \%)\end{array}$ & $\begin{array}{r}27 \\
(4,3 \%)\end{array}$ \\
\hline Insgesamt & $\begin{array}{r}622 \\
(=100 \%) \\
\end{array}$ & $\begin{array}{r}631 \\
(=100 \%) \\
\end{array}$ \\
\hline \multicolumn{3}{|c|}{$\begin{array}{l}1 \text { Aus Gründen der besseren Übersicht wurde auf die Verwendung weiblicher Berufsbezeichnungen } \\
\text { verzichtet. Die Begriffe sind daher geschlechtsneutral zu verstehen. } \\
2 \text { Als Rechtsanwälte und Notare sind nur diejenigen aufgeführt, die tatsächlich auch freiberuflich als } \\
\text { solche tätig waren. Maßgeblich war daher für die Einordnung in die Gruppe 7a nicht die berufliche } \\
\text { Selbstbezeichnung der Abgeordneten, da Rechtsanwälte durchaus auch als Beamte (Gruppe 1), Selb- } \\
\text { ständige (Gruppe 6) oder Angestellte der Wirtschaft (Gruppe 5) tätig sein können. } \\
\text { Quelle: Eigene Zusammenstellung. Die Zahlen für die 17. Wahlperiode stammen aus Melanie Kintz, } \\
\text { Die Berufsstruktur der Abgeordneten des 17. Bundestages, in: ZParl, 41. Jg. (2010), H. 3, S. } 491 \text { - 503, } \\
\text { S. } 493 \text { f. }\end{array}$} \\
\hline
\end{tabular}

Die starken Verschiebungen in den Größenverhältnissen der einzelnen Fraktionen haben zu Veränderungen in der Berufsstruktur geführt (vgl. Tabelle 1). Die Gruppe der Beamten ist zwar auch weiterhin die stärkste Berufsgruppe im Bundestag; ihr Anteil stabilisierte sich mit 29 Prozent (183 Abgeordnete). Innerhalb dieser Berufsgruppe gibt es aber einige erwähnenswerte Veränderungen: Der Anteil der Lehrer ging weiter zurück, weil in dieser Wahlperiode weniger Abgeordnete, die zuvor als Lehrer an Gymnasien (Gruppe 1i) arbeiteten, im Bundestag vertreten sind (13 statt 21). Hingegen stieg der Anteil derer, die zuvor im Bereich der Verwaltung (1b, 1c) tätig waren, leicht an. Besonders auf die Bundestagsneulinge trifft dies zu: 18 der 46 Abgeordneten, die der Gruppe $1 \mathrm{~b}$ angehören, und elf der 21 Parlamentarier der Gruppe 1c sind Neulinge. Ähnlich verhält es sich mit den Kommu- 
nalen Wahlbeamten, deren Anzahl von 30 auf 39 deutlich anstieg. 16 MdBs, die dieser Berufsgruppe zugeordnet werden konnten, sind neu in den Bundestag eingezogen.

Die zweitgrößte Berufsgruppe sind erneut die Angestellten von politischen und gesellschaftlichen Organisationen (Gruppe 4), deren Anteil von 16,6 auf 19,7 Prozent anwuchs. Dies ist wiederum auf einen starken Anstieg in der Gruppe der Angestellten von Parteien und Fraktionen (Gruppe 4a) zurückzuführen. Dieser Untergruppe gehören im 18. Bundestag 73 Abgeordnete an, von denen 22 erstmals in den Bundestag gewählt wurden. Aber auch die beiden anderen Untergruppen, Angestellte von Gewerkschaften und Arbeitnehmerorganisationen (4b) und Angestellte sonstiger Organisationen und Institutionen mit politischen, gesellschaftlichen, kulturellen und karitativen Zielsetzungen (4c), verzeichneten leichte Zuwächse.

Während im 17. Bundestag die 101 Angehörigen der freien Berufe die drittgrößte Berufsgruppe stellten, sind sie mit nunmehr 87 Abgeordneten nur noch die viertstärkste im Bundestag. Der Rückgang ist dabei am deutlichsten in der Gruppe der Rechtsanwälte und Notare zu sehen (von 69 auf 58 Abgeordnete). Auch unter den 217 Bundestagsneulingen ist diese Berufsgruppe weniger stark vertreten, nur 14 (oder 6,5 Prozent) von ihnen gehören ihr an.

Die Angestellten der Wirtschaft (Gruppe 5) sind die neue drittgrößte Berufsgruppe im 18. Bundestag. Gewachsen von 84 auf 93 Mitglieder stellt sie einen Anteil von 14,7 Prozent. Besonders stark ist diese Berufsgruppe unter den neuen Abgeordneten: 42 Neulinge, mithin nahezu jeder fünfte (19,4 Prozent) sind Angestellt der Wirtschaft. Dies steht vermutlich im engen Zusammenhang mit dem guten Wahlergebnis der CDU/CSU-Fraktion.

Vergleicht man die Fraktionen (Tabelle 2), so fallen auch hier starke Unterschiede auf. Wie bereits in der vergangenen Wahlperiode sind die Beamten (Gruppe 1) die größte Berufsgruppe bei den beiden Volksparteien. Allerdings ist ihr Anteil in allen Fraktionen rückläufig. Bei der CDU/CSU stieg zwar die Anzahl der Beamten von 77 auf 97; da diese jedoch viel größer ist als in der 17. Wahlperiode, sank der prozentuale Anteil leicht von 32,2 auf 31,2. In allen anderen Fraktionen sanken sowohl Anzahl als auch prozentualer Anteil der Beamten ${ }^{7}$, am deutlichsten in der SPD (von 43,2 auf 35,8 Prozent). Außerdem fällt auf, dass der bereits erwähnte Zuwachs der Kommunalen Wahlbeamten um insgesamt neun Personen in der Unionsfraktion stattfand, wo $29 \mathrm{MdBs}$ dieser Untergruppe angehören gegenüber 20 in der 17 . Wahlperiode.

Bei den beiden kleineren Fraktionen, Linkspartei und Grüne, sind wie bereits in der vergangenen Wahlperiode die Angestellten von politischen und gesellschaftlichen Organisationen die dominante Berufsgruppe. Bei den Grünen nahm ihre Zahl von 18 auf 24 zu (oder von 26,5 auf 38,1 Prozent). Die Gruppe der Angestellten der Parteien und Fraktionen (Gruppe 4a) verzeichnet mit nun 19 Mitgliedern den größten Zuwachs. Bei der Linkspartei blieb zwar die Zahl der Angestellten von politischen und gesellschaftlichen Organisationen gleich, jedoch nehmen diese in der kleineren Fraktion einen größeren Anteil ein (von 39,5 Prozent in der 17. Wahlperiode auf nunmehr 46,9 Prozent). Aber auch in der SPD gibt es deutlich mehr Fraktionsmitglieder, die aus dieser Berufsgruppe stammen. Während in der vorigen Wahlperiode 29 SPD-Abgeordnete hier einzuordnen waren, sind es in dieser bereits 46 und damit fast jedes vierte Fraktionsmitglied (23,8 Prozent). Besonders stark ist

7 Trotzdem ist diese Berufsgruppe in der Linkspartei zweitstärkste, auch wenn der Anteil rückläufig ist. 
der Anstieg auch hier bei den Angestellten von Parteien und Fraktionen (von 13 auf 21); aber auch bei den Angestellten von Gewerkschaften und Arbeitnehmerorganisationen (Gruppe 4b) erhöhte sich die Anzahl von zehn auf 14. Dies zeigt, dass die verstärkte Rekrutierung von Abgeordneten aus dem eigenen Mitarbeiterkreis ein Trend ist, der nicht mehr nur auf die kleineren Parteien (Grüne, Linke) begrenzt ist.

Auch die Angestellten der privaten Wirtschaft (Gruppe 5), die gesamt die drittstärkste Berufsgruppe im Bundestag ausmachen, sind ungleichmäßig verteilt. Sie spielen kaum eine Rolle in der Berufsstruktur der beiden kleinen Fraktionen, sind aber zweitstärkste (CDU/ CSU) und drittstärkste (SPD) Berufsgruppe in den Fraktionen der Volksparteien. In beiden hat sich ihre Anzahl recht stark erhöht (um 20 auf 63 in der Union und um zehn auf 25 in der SPD). Auch bei den neuen Abgeordneten zeigt sich dieser Trend, denn 41 der

\begin{tabular}{|c|c|c|c|c|c|}
\hline \multicolumn{6}{|c|}{$\begin{aligned} \text { Tabelle 2: } & \text { Berufsstatistik der 18. Wahlperiode nach Fraktionszugehörigkeit (Veränderung } \\
& \text { zur 17. Wahlperiode sowie Spaltenprozent in Klammern) }\end{aligned}$} \\
\hline \multirow{5}{*}{$\begin{array}{l}\text { Abgeordnete insgesamt } \\
\text { CDU/CSU } \\
\text { SPD } \\
\text { Die Linke } \\
\text { Grüne }\end{array}$} & \multirow{5}{*}{\multicolumn{5}{|c|}{\begin{tabular}{|r|}
$631(+9)$ \\
$311(+72)$ \\
$193(+47)$ \\
$64(-12)$ \\
$63(-5)$ \\
\end{tabular}}} \\
\hline & & & & & \\
\hline & & & & & \\
\hline & & & & & \\
\hline & & & & & \\
\hline Die Berufsgruppen & $\begin{array}{l}\text { CDU/ } \\
\text { CSU }\end{array}$ & SPD & Grüne & $\begin{array}{c}\text { Die } \\
\text { Linke }\end{array}$ & $\begin{array}{c}\text { Ins- } \\
\text { gesamt }\end{array}$ \\
\hline \multicolumn{6}{|l|}{ 1. Beamte } \\
\hline a) So genannte politische Beamte & 4 & 4 & 1 & 0 & 9 \\
\hline $\begin{array}{l}\text { b) Andere Beamte des höheren Dienstes } \\
\text { (Verwaltung) }\end{array}$ & 22 & 21 & 2 & 1 & 46 \\
\hline $\begin{array}{l}\text { c) Beamte des gehobenen und mittleren } \\
\text { Dienstes (Verwaltung) }\end{array}$ & 11 & 7 & 2 & 1 & 21 \\
\hline d) Richter und Staatsanwälte & 2 & 1 & 0 & 1 & 4 \\
\hline e) Berufssoldaten & 2 & 0 & 0 & 0 & 2 \\
\hline f) Kommunale Wahlbeamte & 29 & 8 & 0 & 2 & 39 \\
\hline $\begin{array}{l}\text { g) Professoren an Universitäten und Hoch- } \\
\text { schulen }\end{array}$ & 5 & 3 & 0 & 0 & 8 \\
\hline $\begin{array}{l}\text { h) Andere Wissenschaftler an Universitäten } \\
\text { und Hochschulen }\end{array}$ & 12 & 7 & 3 & 2 & 24 \\
\hline i) Lehrer an Gymnasien (u.ä.) & 6 & 7 & 0 & 0 & 13 \\
\hline $\begin{array}{l}\text { j) Lehrer an Grund-, Hauptschulen und } \\
\text { Realschulen (u.ä.) }\end{array}$ & 4 & 11 & 1 & 1 & 17 \\
\hline \multirow{2}{*}{ Beamte zusammen } & $\begin{array}{r}97 \\
(+20)\end{array}$ & $\begin{array}{r}69 \\
(-6)\end{array}$ & $\begin{array}{r}9 \\
(-3)\end{array}$ & $\begin{array}{r}8 \\
(-6)\end{array}$ & $\begin{array}{l}183 \\
(-1)\end{array}$ \\
\hline & $(31,2 \%)$ & $(35,8 \%)$ & $(14,3 \%)$ & $(12,5 \%)$ & $(29,0 \%)$ \\
\hline $\begin{array}{l}\text { 2. Angestellte des öffentlichen Dienstes } \\
\text { (auch von Körperschaften, Anstalten und }\end{array}$ & $\begin{array}{r}8 \\
(-2)\end{array}$ & $\begin{array}{r}6 \\
(-5)\end{array}$ & $\begin{array}{r}2 \\
(-2)\end{array}$ & $\begin{array}{r}4 \\
(0)\end{array}$ & $\begin{array}{r}20 \\
(-11)\end{array}$ \\
\hline $\begin{array}{l}\text { Stiftungen des öffentlichen Rechts) zusam- } \\
\text { men }\end{array}$ & $(2,6 \%)$ & $(3,1 \%)$ & $(3,2 \%)$ & $(6,3 \%)$ & $(3,2 \%)$ \\
\hline a) Bedienstete der EG/EU zusammen & 1 & 0 & 0 & 0 & 1 \\
\hline 3. (Ev.) Pfarrer und Diakone zusammen & $\begin{array}{r}1 \\
(-1) \\
(0,3 \%) \\
\end{array}$ & $\begin{array}{r}1 \\
(+1) \\
(0,5 \%)\end{array}$ & $\begin{array}{r}0 \\
(-1)\end{array}$ & $\begin{array}{r}0 \\
(0)\end{array}$ & $\begin{array}{r}2 \\
(-1) \\
(0,3 \%) \\
\end{array}$ \\
\hline
\end{tabular}




\begin{tabular}{|c|c|c|c|c|c|}
\hline \multicolumn{6}{|l|}{ Fortsetzung Tabelle 2} \\
\hline \multirow{3}{*}{$\begin{array}{l}\text { 4. Angestellte von politischen und gesell- } \\
\text { schaftlichen Organisationen } \\
\text { a) Angestellte von Parteien, Fraktionen (u.ä.) } \\
\text { b) Angestellte von Gewerkschaften und Ar- } \\
\text { beitnehmerorganisationen } \\
\text { c) Angestellte sonstiger Organisationen und } \\
\text { Institutionen mit politischen, gesellschaft- } \\
\text { lichen, kulturellen und karitativen Zielset- } \\
\text { zungen }\end{array}$} & 16 & 21 & 19 & 17 & 73 \\
\hline & 0 & 14 & 0 & 8 & 22 \\
\hline & 8 & 11 & 5 & 5 & 29 \\
\hline \multirow{3}{*}{ Angestellte dieser Organisationen zusammen } & 24 & & 24 & 30 & 124 \\
\hline & $(+3)$ & $(+17)$ & $(+6)$ & $(0)$ & $(+21)$ \\
\hline & $(7,7 \%)$ & $(23,8 \%)$ & $(38,1 \%)$ & $(46,9 \%)$ & $(19,7 \%)$ \\
\hline \multirow{3}{*}{$\begin{array}{l}\text { 5. Angestellte der Wirtschaft } \\
\text { (in Industrie, Handel, Handwerk, Gewerbe, } \\
\text { und entsprechenden Verbänden) zusammen }\end{array}$} & 63 & & 4 & 1 & 93 \\
\hline & $(+20)$ & $(+10)$ & $(-1)$ & $(-3)$ & $(+10)$ \\
\hline & $(20,3 \%)$ & $(13,0 \%)$ & $(6,4 \%)$ & $(1,6 \%)$ & $(14,7 \%)$ \\
\hline \multirow{4}{*}{$\begin{array}{l}\text { 6. Selbständige } \\
\text { (oft zugleich in entsprechenden Verbänden } \\
\text { tätig) } \\
\text { a) Selbständige in Industrie, Handel Hand- } \\
\text { werk und Gewerbe } \\
\text { b) Selbständige in Land- und Forstwirtschaft }\end{array}$} & & & & & \\
\hline & 33 & 9 & 3 & 1 & 46 \\
\hline & 14 & 0 & 1 & 0 & 15 \\
\hline & & & & & 61 \\
\hline \multirow[t]{2}{*}{ Selbständige zusammen } & $(+10)$ & $(+8)$ & $(+2)$ & $(-1)$ & $(+1)$ \\
\hline & $(15,1 \%)$ & $(4,7 \%)$ & $(6,4 \%)$ & $(1,6 \%)$ & $(9,7 \%)$ \\
\hline \multirow{3}{*}{$\begin{array}{l}\text { 7. Angehörige freier Berufe } \\
\text { a) Rechtsanwälte und Notare } \\
\text { b) Angehörige anderer freier Berufe } \\
\text { (Ärzte, Apotheker, Ingenieure, Steuer- } \\
\text { berater, Schriftsteller, Journalisten u.ä.) }\end{array}$} & 38 & 13 & 4 & 3 & 58 \\
\hline & 11 & 10 & 7 & 1 & 29 \\
\hline & 49 & 23 & 11 & 4 & 87 \\
\hline \multirow{2}{*}{ Freiberufler zusammen } & $(+11)$ & $(+6)$ & $(\rightarrow)$ & $(-2)$ & $(-14)$ \\
\hline & $(15,8 \%)$ & $(11,9 \%)$ & $(17,5 \%)$ & $(6,3 \%)$ & $(13,8 \%)$ \\
\hline \multirow[t]{2}{*}{ 8. Hausfrauen zusammen } & $\begin{array}{r}1 \\
(0)\end{array}$ & $\begin{array}{r}3 \\
(+1)\end{array}$ & $\begin{array}{r}0 \\
(0)\end{array}$ & $\begin{array}{r}0 \\
(0)\end{array}$ & $\begin{array}{r}4 \\
(+1)\end{array}$ \\
\hline & $(0,3 \%)$ & $(1,6 \%)$ & & & $(0,6 \%)$ \\
\hline $\begin{array}{l}\text { 9. Arbeiter zusammen } \\
\text { (ohne solche Abgeordnete, die zwar beruflich } \\
\text { als Arbeiter begonnen haben, jetzt aber ande- } \\
\text { ren Gruppen zuzurechnen sind) }\end{array}$ & $\begin{array}{r}0 \\
(0)\end{array}$ & $\begin{array}{r}0 \\
(-1)\end{array}$ & $\begin{array}{r}0 \\
(0)\end{array}$ & $\begin{array}{r}1 \\
(0) \\
(1,6 \%)\end{array}$ & $\begin{array}{r}1 \\
(-1) \\
(0,2 \%) \\
\end{array}$ \\
\hline \multirow{2}{*}{$\begin{array}{l}\text { 10. Sonstige zusammen } \\
\text { (darunter in Ausbildung befindliche, Arbeits- } \\
\text { lose oder bisher ohne Berufsausübung) }\end{array}$} & $\begin{array}{r}12 \\
(+5)\end{array}$ & $\begin{array}{r}2 \\
(-1)\end{array}$ & $\begin{array}{r}7 \\
(+2)\end{array}$ & $\begin{array}{r}7 \\
(-1)\end{array}$ & $\begin{array}{r}28 \\
(+1)\end{array}$ \\
\hline & $(4,5 \%)$ & $(1,0 \%)$ & $(11,1 \%)$ & $(10,9 \%)$ & $(4,4 \%)$ \\
\hline \multirow{2}{*}{$\begin{array}{l}\text { 11. Nicht verwendbare Angaben } \\
\text { (fehlende genaue Angaben im Sinne des } \\
\text { orliegenden Schemas) }\end{array}$} & $\begin{array}{r}9 \\
(+5)\end{array}$ & $\begin{array}{r}9 \\
(+5)\end{array}$ & $\begin{array}{r}2 \\
(-3)\end{array}$ & $\begin{array}{r}8 \\
(+1)\end{array}$ & $\begin{array}{r}28 \\
(+4)\end{array}$ \\
\hline & $(2,9 \%)$ & $(4,7 \%)$ & $(3,2 \%)$ & $(12,5 \%)$ & $(4,4 \%)$ \\
\hline Insgesamt & 311 & 193 & 63 & 64 & 631 \\
\hline
\end{tabular}


42 Bundestagsneulinge, die dieser Berufsgruppe entstammen, gehören der CDU/CSU(27) oder der SPD-Fraktion (14) an.

Die Freiberufler sind die zweitstärkste Berufsgruppe bei den Grünen (elf Abgeordnete) und die drittstärkste in der Unionsfraktion (49 Abgeordnete). Während dabei in der CDU/ CSU der Anteil der Anwälte und Notare (Gruppe 7a) größer ist (38 von 49 Abgeordneten), sind es bei den Grünen die Angehörigen anderer freier Berufe (Gruppe 7b), die den größeren Anteil einnehmen (sieben von elf Abgeordneten). Dennoch ist das Schrumpfen dieser Berufsgruppe hauptsächlich auf das Ausscheiden der FDP aus dem Parlament zurückzuführen. In ihrer 93 Abgeordnete starken Fraktion des 17. Bundestages waren 26,1 Prozent der Mitglieder Freiberufler.

Wie bereits in der vergangenen Wahlperiode gehören dem Bundestag auch wieder 30 Abgeordnete an, die in der Gruppe „Sonstige“ zusammengefasst werden. Diese besteht hauptsächlich aus Studenten. In der Fraktion der Linkspartei bilden sie die drittstärkste Gruppe. ${ }^{8}$ Zeigt sich hier also ein starkes Rekrutierungspotenzial für die Linke, so ist in diesem Zusammenhang auch bemerkenswert, dass diese Gruppe innerhalb der CDU/CSUFraktion einen deutlichen Zuwachs von sieben auf zwölf Mitglieder erfahren hat. Unter den sechs Bundestagsneulingen, die den „Sonstigen“ zuzuordnen wären, stammen vier aus der Unionsfraktion. ${ }^{9}$

\section{Berufsstruktur ostdeutscher und westdeutscher Abgeordneter: Entwicklungstendenzen}

Da die vorangegangenen Untersuchungen gezeigt haben, dass auch mehr als 20 Jahre nach der Wiedervereinigung noch deutliche Unterschiede in der Berufsstruktur ost- und westdeutscher Abgeordneter zu sehen sind, scheint es sinnvoll, diesen Vergleich fortzuführen. In der 18. Wahlperiode sind 92 Ostdeutsche im Bundestag vertreten. Damit stabilisiert sich deren Anteil seit dem 16. Bundestag auf einem relativ niedrigen Niveau von 14,6 (18. Bundestag) bis 15,1 Prozent (16. Bundestag). Die Fraktion mit dem größten Anteil ostdeutscher Abgeordneter in ihren Reihen ist nach wie vor die Linkspartei mit 45,3 Prozent. Während Ostdeutsche damit in der Linkspartei verglichen zum Anteil an der Bevölkerung überrepräsentiert sind, sind sie in allen anderen Fraktionen unterrepräsentiert. In der jetzt viel größeren Unionsfraktion sind 41 ostdeutsche Abgeordnete vertreten, die einen Anteil von 13,2 Prozent ausmachen und damit einen leichten Anstieg gegenüber den beiden vorangegangenen Wahlperioden bedeuten. In der SPD-Fraktion stieg die Zahl der Ostdeutschen um zwei auf 16; ihr Anteil schrumpfte hingegen auf 8,3 Prozent, da sich die Fraktion mit der letzten Bundestagswahl deutlich vergrößerte. Unter den 217 neugewählten Abgeordneten sind auch anteilig weniger ostdeutsche, nämlich lediglich zwölf Prozent.

8 Auch wenn die Gruppe derer, deren Berufsbezeichnung nicht im Sinne des Kategorienschemas verwendbar ist, etwas größer ist (Gruppe 11).

9 Der fünfte Abgeordnete, der neu dieser Gruppe zugeordnet wurde, war bereits im vergangenen Bundestag vertreten. Allerdings konnte er zum Zeitpunkt der Publikation des Artikels für die 17. Wahlperiode nicht eindeutig einer Berufsgruppe zugeordnet werden und fiel in die Gruppe 11. Durch weitere Informationen, die jetzt verfügbar waren, konnte er der Gruppe 10 zugeordnet werden. 
Die meisten (79) ostdeutschen MdBs wurden über Listen und Wahlkreise der neuen Bundesländer oder der Stadt Berlin (7) ins Parlament gewählt. Aber sechs Abgeordnete, die in der DDR aufgewachsen sind, wurden über Listen und in Wahlkreisen der alten Bundesländer gewählt, davon erstmalig auch jeweils ein Mitglied der Fraktion von CDU/CSU, Grüne und SPD. ${ }^{10}$ Im Gegenzug gelang es 22 Abgeordneten westdeutscher Herkunft, über Listen und Wahlkreise in den neuen Bundesländern in den Bundestag einzuziehen.

Betrachtet man die Berufsstruktur der beiden Gruppen (vgl. Tabelle 3), so fallen im Vergleich zu den vorherigen Wahlperioden nur wenige Veränderungen auf: Die bestehenden Unterschiede verfestigen sich also. In beiden Gruppen ist auch im 18. Bundestag die Gruppe der Beamten die stärkste, auch wenn ihr prozentual gesehen weniger Ostdeutsche angehören (27,2 Prozent) als Westdeutsche (29,8 Prozent). Hier gibt der Unterkategorienvergleich eher Aufschluss. Unter den ostdeutschen Abgeordneten gibt es keine, die vor ihrem Bundestagsmandat als politische Beamte (1a), Richter und Staatsanwälte (1d), Berufssoldaten (1e), Professoren (1g) oder Lehrer an Gymnasien (1i) tätig waren. Zum Teil entspricht dies vermutlich den Berufsgruppen, in denen Ostdeutsche generell noch unterrepräsentiert sind. ${ }^{11}$ Aber auch die geringeren Verbeamtungsraten in den neuen Bundesländern dürften als Ursache zu sehen sein. Sind ostdeutsche MdBs Beamte, dann kommen sie häufiger als ihre westdeutschen Kollegen aus der Gruppe der Kommunalen Wahlbeamten (1f) und der Wissenschaftler (1h). Zweitstärkste Berufsgruppe sind bei Ost- wie Westdeutschen die Angestellten politischer Organisationen, wobei dies mehr für ostdeutsche Parlamentarier (22,8 Prozent) als für westdeutsche (19,2 Prozent) gilt. Verglichen mit den vorherigen Wahlperioden scheint der Anteil dieser Berufsgruppe unter den ostdeutschen Abgeordneten schneller anzusteigen als bei den westdeutschen, was vermutlich auf die stärkere Rekrutierung der Linkspartei aus diesem Sektor zurückzuführen ist. Besonders bemerkenswert ist hierbei auch, dass Ostdeutsche hauptsächlich der Gruppe der Angestellten der Parteien und Fraktionen entstammen (81,0 Prozent derer, die der Gruppe 4 zuzuordnen sind), während dies auf nur etwas mehr als die Hälfte (54,5 Prozent) der Westdeutschen zutrifft. Diese sind stärker unter den Angestellten von Gewerkschaften und Arbeitnehmerorganisationen (Gruppe 4b) sowie anderer Gesellschaftlicher Organisationen (4c) vertreten.

Bei den westdeutschen Abgeordneten ist die Gruppe der Freiberufler die drittstärkste (15,4 Prozent); hingegen gehören nur vier ostdeutsche Parlamentarier (4,4 Prozent) ihr an. Seit der ersten Erhebung des Ost-West-Vergleichs zeigt sich dies als wiederkehrendes Muster. Die drittgrößte Berufsgruppe bei den ostdeutschen MdBs bilden mit 14 Personen (15,2 Prozent) die Angestellten der Privatwirtschaft (Gruppe 5),

Während in der Anfangszeit nach der Wiedervereinigung mehr ostdeutsche Abgeordnete ihren beruflichen Hintergrund im kirchlichen Milieu (Gruppe 3) hatten, ist dieser Unterschied jetzt gänzlich verschwunden. Dies zeigt, dass die „Übergangselite“ fast vollständig

10 Wie bereits in der vergangenen Wahlperiode zogen drei Abgeordnete der Linkspartei über Listen in den alten Bundesländern in den Bundestag ein.

11 Neuere Daten sind hierzu kaum erhältlich, die aktuellsten Informationen gehen zurück auf Christoph Cadenbach / Bastian Obermaier, Geschlossene Gesellschaft, in: SZ-Magazin vom 30. Juli 2010, http://sz-magazin.sueddeutsche.de/texte/anzeigen/34445/Geschlossene-Gesellschaft (Abruf am 12. August 2014). 


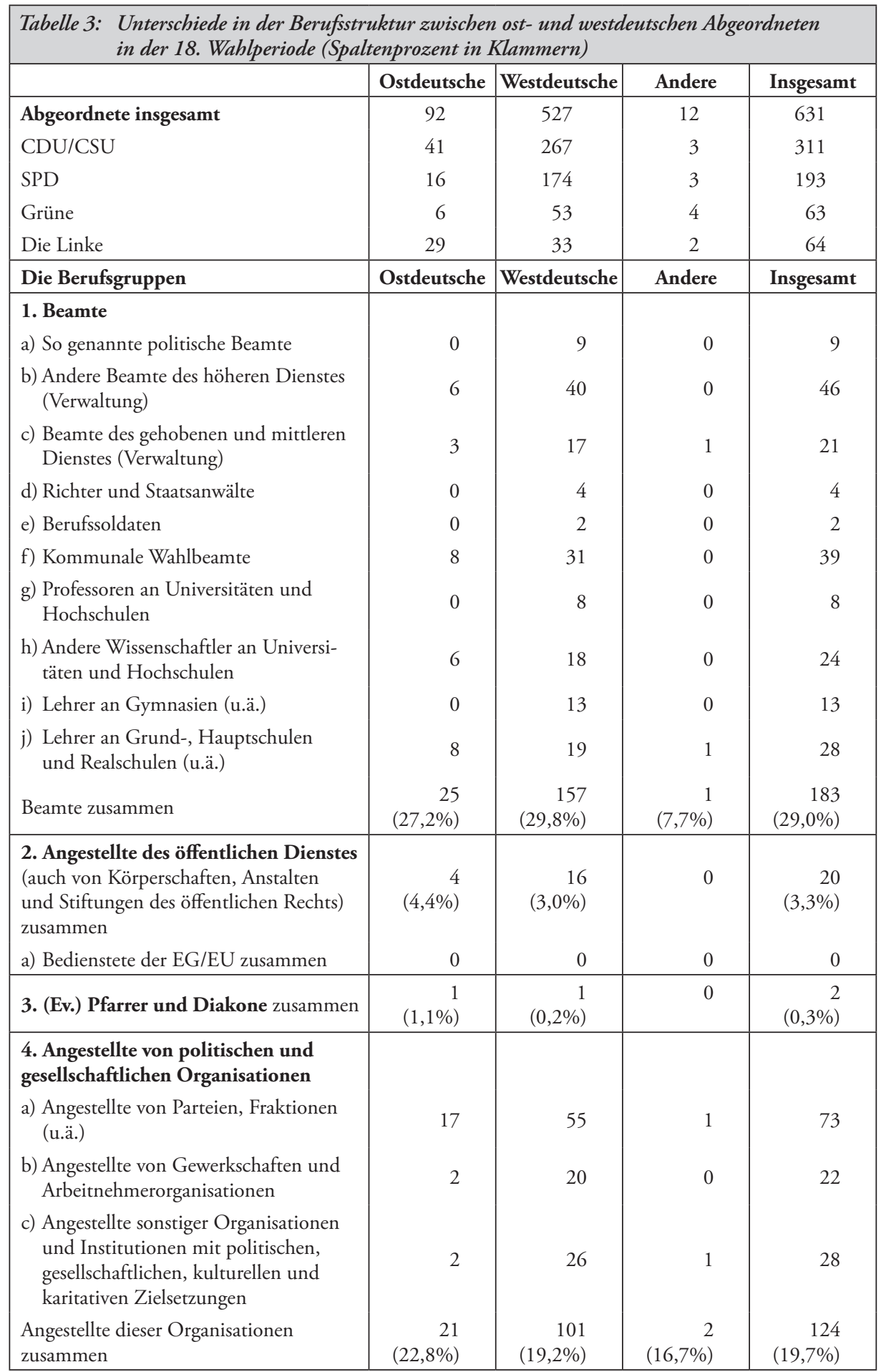




\begin{tabular}{|c|c|c|c|c|}
\hline \multicolumn{5}{|l|}{ Fortsetzung Tabelle 3} \\
\hline $\begin{array}{l}\text { 5. Angestellte der Wirtschaft } \\
\text { (in Industrie, Handel, Handwerk, } \\
\text { Gewerbe, und entsprechenden } \\
\text { Verbänden) zusammen }\end{array}$ & $\begin{array}{r}14 \\
(15,2 \%)\end{array}$ & $\begin{array}{r}77 \\
(14,6 \%)\end{array}$ & $\begin{array}{r}2 \\
(16,7 \%)\end{array}$ & $\begin{array}{r}93 \\
(14,7 \%)\end{array}$ \\
\hline \multicolumn{5}{|l|}{$\begin{array}{l}\text { 6. Selbständige } \\
\text { (oft zugleich in entsprechenden } \\
\text { Verbänden tätig) }\end{array}$} \\
\hline $\begin{array}{l}\text { a) Selbständige in Industrie, Handel } \\
\text { Handwerk und Gewerbe }\end{array}$ & 7 & 37 & 2 & 46 \\
\hline $\begin{array}{l}\text { b) Selbständige in Land- und } \\
\text { Forstwirtschaft }\end{array}$ & 0 & 15 & 0 & 15 \\
\hline Selbständige zusammen & $\begin{array}{r}7 \\
(7,6 \%)\end{array}$ & $\begin{array}{r}52 \\
(9,9 \%) \\
\end{array}$ & $\begin{array}{r}1 \\
(16,7 \%) \\
\end{array}$ & $\begin{array}{r}61 \\
(9,6 \%) \\
\end{array}$ \\
\hline \multicolumn{5}{|l|}{ 7. Angehörige freier Berufe } \\
\hline a) Rechtsanwälte und Notare & 3 & 53 & 2 & 58 \\
\hline $\begin{array}{l}\text { b) Angehörige anderer freier Berufe } \\
\text { (Ärzte, Apotheker, Ingenieure, Steuer- } \\
\text { berater, Schriftsteller, Journalisten u.ä.) }\end{array}$ & 1 & 28 & 0 & 29 \\
\hline Freiberufler zusammen & $\begin{array}{r}4 \\
(4,6 \%) \\
\end{array}$ & $\begin{array}{r}81 \\
(15,4 \%) \\
\end{array}$ & $\begin{array}{r}2 \\
(16,7 \%) \\
\end{array}$ & $\begin{array}{r}87 \\
(13,8 \%) \\
\end{array}$ \\
\hline 8. Hausfrauen zusammen & 0 & $\begin{array}{r}4 \\
(0,8 \%) \\
\end{array}$ & 0 & $\begin{array}{r}4 \\
(0,6 \%) \\
\end{array}$ \\
\hline $\begin{array}{l}\text { 9. Arbeiter zusammen } \\
\text { (ohne solche Abgeordnete, die zwar } \\
\text { beruflich als Arbeiter begonnen haben, } \\
\text { jetzt aber anderen Gruppen zuzurechnen } \\
\text { sind) }\end{array}$ & 0 & $\begin{array}{r}1 \\
(0,2 \%)\end{array}$ & 0 & $\begin{array}{r}1 \\
(0,2 \%)\end{array}$ \\
\hline $\begin{array}{l}\text { 10. Sonstige zusammen } \\
\text { (darunter in Ausbildung befindliche, } \\
\text { Arbeitslose oder bisher ohne Berufsaus- } \\
\text { übung) }\end{array}$ & $\begin{array}{r}8 \\
(8,7 \%)\end{array}$ & $\begin{array}{r}18 \\
(3,4 \%)\end{array}$ & $\begin{array}{r}2 \\
(16,7 \%)\end{array}$ & $\begin{array}{r}28 \\
(4,4 \%)\end{array}$ \\
\hline $\begin{array}{l}\text { 11. Nicht verwendbare Angaben } \\
\text { (fehlende genaue Angaben im Sinne } \\
\text { des vorliegenden Schemas) }\end{array}$ & $\begin{array}{r}8 \\
(8,7 \%)\end{array}$ & $\begin{array}{r}19 \\
(3,6 \%)\end{array}$ & $\begin{array}{r}1 \\
(8,3 \%)\end{array}$ & $\begin{array}{r}28 \\
(4,4 \%)\end{array}$ \\
\hline Insgesamt & 92 & 527 & 12 & 631 \\
\hline
\end{tabular}

aus dem Bundestag ausgeschieden ist. Auch sitzen nur noch fünf Mitglieder der letzten DDR-Volkskammer im Bundestag, wobei mit Kerstin Kassner (Linkspartei) auch ein Bundestagsneuling darunter ist. Insgesamt gibt es sieben ostdeutsche Parlamentarier der „ersten Stunde" 12 , inklusive der Kanzlerin Angela Merkel (CDU) und der neuen Ostbeauftragten Iris Gleicke (SPD).

12 Das sind Bundestagsabgeordnete, die seit 1990 im Bundestag sind. 


\section{Entwicklungstendenzen in der Berufsstruktur jüngerer Abgeordneter}

Um Aussagen treffen zu können, ob sich die Berufsstrukturen ost- und westdeutscher Abgeordneter angleichen, und neue Entwicklungstrends zu identifizieren, ist es hilfreich, die jüngeren Parlamentarier genauer zu betrachten. Dafür werden hier alle MdBs herangezogen, die 201340 Jahre alt oder jünger waren. Obwohl der 18. Bundestag mit 34,6 Prozent eine überdurchschnittlich hohe Erneuerungsrate aufweist, ist die Anzahl der jungen Abgeordneten kleiner (125) als in der letzten Wahlperiode (133). Mehr als die Hälfte (72 Abgeordnete, 57,6 Prozent) sind Bundestagsneulinge. Die meisten (66) jungen Abgeordneten sind in der CDU/CSU-Fraktion zu finden, und von ihnen sind 40 erstmals in den Bundestag eingezogen. In der SPD-Fraktion, der 30 junge Abgeordnete angehören, sind es gar 22 Neulinge. Bei den Grünen sind neun der 19, bei der Linkspartei nur einer der zehn jungen Abgeordneten zum ersten Mal im Parlament. Durch den starken Zufluss neuer Abgeordneter sind auch Verschiebungen in der Ost-West-Verteilung aufgetreten (vgl. Tabelle 4). Gehörten in der letzten Wahlperiode die meisten ostdeutschen jungen Abgeordneten der Fraktion der Linkspartei an, sind jetzt zwölf der 24 jungen Ostdeutschen in der Unionsfraktion zu finden, die auch 54,5 Prozent der westdeutschen jungen Abgeordneten in ihren Reihen hat. Die zweitgrößte Gruppe (26,3 Prozent) der jungen Westdeutschen ist in der SPD-Fraktion, die zweitstärkste Gruppe der jungen Ostdeutschen in der Fraktion der Linken beheimatet.

Gesamt gesehen unterscheidet sich die Berufsstruktur der jungen Abgeordneten von der des gesamten Bundestags darin, dass die Angestellten politischer Organisationen (Gruppe 4) und die Angestellten der Wirtschaft (Gruppe 5) die größten Gruppen sind (mit jeweils 27 Abgeordneten, 21,6 Prozent), während die Gruppe der Beamten (Gruppe 1) den dritten Platz einnimmt (20,8 Prozent). Dies lässt vermuten, dass die Herkunft der Parlamentarier aus der Beamtenschaft weiter an Bedeutung verlieren wird.

Auch beim Ost-West-Vergleich zeigen sich noch deutliche Unterschiede, allerdings ist bei der Auswertung zu beachten, dass die Ergebnisse auf recht kleinen Zahlen beruhen. Betrachtet man die beiden größten Berufsgruppen, fällt auf, dass die ostdeutschen jungen Abgeordneten viel häufiger aus der Gruppe der Angestellten politischer Organisationen kommen (Gruppe 4; 29,2 Prozent vs. 19,1 Prozent). Dafür sind ihre westdeutschen Altersgenossen viel öfter Angestellte der Wirtschaft gewesen (Gruppe 5; 24,2 vs. 12,5 Prozent). Auch der Gruppe der Beamten entstammt ein größerer Teil westdeutscher junger Abgeordneter (23,2 Prozent) als ostdeutscher (12,5 Prozent). Letztere sind stärker in der Gruppe der Sonstigen (Gruppe 10) vertreten. Der Befund des allgemeinen Ost-West-Vergleichs (Tabelle 3), dass Ostdeutsche seltener selbstständig (Gruppe 6) und freiberuflich (Gruppe 7) tätig sind, findet sich weniger stark ausgeprägt bei den jungen Abgeordneten. Allerdings sind die Zahlen hier zu klein, um verlässliche Aussagen zu treffen. Gesamt gesehen zeigen sich auch bei den jungen Abgeordneten noch deutliche Unterschiede in den Berufsstrukturen, die sich nicht nur mit ihrer unterschiedlichen Verteilung auf die Fraktionen erklären lassen.

\section{Ein Trend zu mehr politiknahen Vorberufen?}

Obwohl 2013 überdurchschnittlich viele Neulinge in den Bundestag einzogen und die FDP an der Fünf-Prozent-Hürde scheiterte, gab es in der Berufsstruktur der deutschen Parlamentarier nur wenig bedeutungsvolle Veränderungen. Im Ost-West-Vergleich sind An- 


\begin{tabular}{|c|c|c|c|c|}
\hline \multicolumn{5}{|c|}{$\begin{aligned} \text { Tabelle 4: Unterschiede in der Berufsstruktur zwischen ost- und westdeutschen Abgeordneten im } \\
\text { Alter bis einschließlich 40 Jahre in der 18. Wahlperiode (Spaltenprozent in Klammern) }\end{aligned}$} \\
\hline & Ostdeutsche & Westdeutsche & Andere & Insgesamt \\
\hline Abgeordnete insgesamt & 24 & 99 & 2 & 125 \\
\hline CDU/CSU & 12 & 54 & 0 & 66 \\
\hline SPD & 4 & 26 & 0 & 30 \\
\hline Grüne & 2 & 15 & 2 & 19 \\
\hline Die Linke & 6 & 4 & 0 & 10 \\
\hline Die Berufsgruppen & Ostdeutsche & Westdeutsche & Andere & Insgesamt \\
\hline 1. Beamte & & & & \\
\hline a) So genannte politische Beamte & 0 & 0 & 0 & 0 \\
\hline $\begin{array}{l}\text { b) Andere Beamte des höheren Dienstes } \\
\text { (Verwaltung) }\end{array}$ & 1 & 6 & 0 & 7 \\
\hline $\begin{array}{l}\text { c) Beamte des gehobenen und mittleren } \\
\text { Dienstes (Verwaltung) }\end{array}$ & 0 & 6 & 0 & 6 \\
\hline d) Richter und Staatsanwälte & 0 & 1 & 0 & 1 \\
\hline e) Berufssoldaten & 0 & 0 & 0 & 0 \\
\hline f) Kommunale Wahlbeamte & 0 & 3 & 0 & 3 \\
\hline $\begin{array}{l}\text { g) Professoren an Universitäten und } \\
\text { Hochschulen }\end{array}$ & 0 & 1 & 0 & 1 \\
\hline $\begin{array}{l}\text { h) Andere Wissenschaftler an Universi- } \\
\text { täten und Hochschulen }\end{array}$ & 2 & 4 & 0 & 6 \\
\hline i) Lehrer an Gymnasien (u.ä.) & 0 & 2 & 0 & 2 \\
\hline $\begin{array}{l}\text { j) Lehrer an Grund-, Hauptschulen } \\
\text { und Realschulen (u.ä.) }\end{array}$ & 0 & 0 & 0 & 0 \\
\hline Beamte zusammen & $\begin{array}{r}3 \\
(12,5 \%) \\
\end{array}$ & $\begin{array}{r}23 \\
(23,2 \%)\end{array}$ & 0 & $\begin{array}{r}26 \\
(20,8 \%) \\
\end{array}$ \\
\hline $\begin{array}{l}\text { 2. Angestellte des öffentlichen Dienstes } \\
\text { (auch von Körperschaften, Anstalten } \\
\text { und Stiftungen des öffentlichen Rechts) } \\
\text { zusammen }\end{array}$ & 0 & 0 & 0 & 0 \\
\hline a) Bedienstete der EG/EU zusammen & 0 & 0 & 0 & 0 \\
\hline 3. (Ev.) Pfarrer und Diakone zusammen & 0 & 0 & 0 & 0 \\
\hline $\begin{array}{l}\text { 4. Angestellte von politischen und } \\
\text { gesellschaftlichen Organisationen }\end{array}$ & & & & \\
\hline $\begin{array}{l}\text { a) Angestellte von Parteien, Fraktionen } \\
\text { (u.ä.) }\end{array}$ & 6 & 16 & 1 & 23 \\
\hline $\begin{array}{l}\text { b) Angestellte von Gewerkschaften und } \\
\text { Arbeitnehmerorganisationen }\end{array}$ & 0 & 0 & 0 & 0 \\
\hline $\begin{array}{l}\text { c) Angestellte sonstiger Organisationen } \\
\text { und Institutionen mit politischen, } \\
\text { gesellschaftlichen, kulturellen und } \\
\text { karitativen Zielsetzungen }\end{array}$ & 1 & 2 & 0 & 3 \\
\hline $\begin{array}{l}\text { Angestellte dieser Organisationen } \\
\text { zusammen }\end{array}$ & $\begin{array}{r}7 \\
(29,2 \%) \\
\end{array}$ & $\begin{array}{r}19 \\
(19,2 \%) \\
\end{array}$ & $\begin{array}{r}1 \\
(50,0 \%) \\
\end{array}$ & $\begin{array}{r}27 \\
(21,6 \%) \\
\end{array}$ \\
\hline $\begin{array}{l}\text { 5. Angestellte der Wirtschaft } \\
\text { (in Industrie, Handel, Handwerk, } \\
\text { Gewerbe, und entsprechenden } \\
\text { Verbänden) zusammen } \\
\end{array}$ & $\begin{array}{r}3 \\
(12,5 \%)\end{array}$ & $\begin{array}{r}24 \\
(24,2 \%)\end{array}$ & 0 & $\begin{array}{r}27 \\
(21,6 \%)\end{array}$ \\
\hline
\end{tabular}




\begin{tabular}{|c|c|c|c|c|}
\hline \multicolumn{5}{|l|}{ Fortsetzung Tabelle 4} \\
\hline $\begin{array}{l}\text { 6. Selbständige } \\
\text { (oft zugleich in entsprechenden } \\
\text { Verbänden tätig) }\end{array}$ & & & & \\
\hline $\begin{array}{l}\text { a) Selbständige in Industrie, Handel } \\
\text { Handwerk und Gewerbe }\end{array}$ & 1 & 2 & 0 & 3 \\
\hline $\begin{array}{l}\text { b) Selbständige in Land- und Forst- } \\
\text { wirtschaft }\end{array}$ & 0 & 1 & 0 & 1 \\
\hline Selbständige zusammen & $\begin{array}{r}1 \\
(4,2 \%) \\
\end{array}$ & $\begin{array}{r}3 \\
(3,0 \%)\end{array}$ & 0 & $\begin{array}{r}4 \\
(3,2 \%) \\
\end{array}$ \\
\hline $\begin{array}{l}\text { 7. Angehörige freier Berufe } \\
\text { a) Rechtsanwälte und Notare }\end{array}$ & 2 & 11 & 0 & 13 \\
\hline $\begin{array}{l}\text { b) Angehörige anderer freier Berufe } \\
\text { (Ärzte, Apotheker, Ingenieure, Steuer- } \\
\text { berater, Schriftsteller, Journalisten u.ä.) }\end{array}$ & 0 & 2 & 0 & 2 \\
\hline Freiberufler zusammen & $\begin{array}{r}2 \\
(8,3 \%) \\
\end{array}$ & $\begin{array}{r}13 \\
(13,1 \%) \\
\end{array}$ & 0 & $\begin{array}{r}15 \\
(12,0 \%) \\
\end{array}$ \\
\hline 8. Hausfrauen zusammen & 0 & 0 & 0 & 0 \\
\hline $\begin{array}{l}\text { 9. Arbeiter zusammen } \\
\text { (ohne solche Abgeordnete, die zwar beruf- } \\
\text { lich als Arbeiter begonnen haben, jetzt } \\
\text { aber anderen Gruppen zuzurechnen sind) }\end{array}$ & 0 & 0 & 0 & 0 \\
\hline $\begin{array}{l}\text { 10. Sonstige zusammen } \\
\text { (darunter in Ausbildung befindliche, } \\
\text { Arbeitslose oder bisher ohne Berufsaus- } \\
\text { übung) }\end{array}$ & $\begin{array}{r}5 \\
(20,8 \%)\end{array}$ & $\begin{array}{r}14 \\
(14,1 \%)\end{array}$ & $\begin{array}{r}1 \\
(50,0 \%)\end{array}$ & $\begin{array}{r}20 \\
(16,0 \%)\end{array}$ \\
\hline $\begin{array}{l}\text { 11. Nicht verwendbare Angaben } \\
\text { (fehlende genaue Angaben im Sinne } \\
\text { des vorliegenden Schemas) }\end{array}$ & $\begin{array}{r}3 \\
(12,5 \%)\end{array}$ & $\begin{array}{r}3 \\
(3,0 \%)\end{array}$ & 0 & $\begin{array}{r}6 \\
(4,8 \%)\end{array}$ \\
\hline Insgesamt & 24 & 99 & 2 & 125 \\
\hline
\end{tabular}

gleichungstendenzen zu beobachten, allerdings bestehen weiterhin einige deutliche Unterschiede in der Berufsstruktur (speziell in den Gruppen der Freiberufler und Beamten), die sich auch unter den jungen Abgeordneten wiederfinden. Des Weiteren gibt es im 18. Bundestag weniger Abgeordnete, die ihrem Vorberuf nach der Gruppe der Freiberufler zuzuordnen sind.

Bedeutsamer erscheinen der Anstieg der Kommunalen Wahlbeamten unter den Beamten und der erneute Zuwachs der Angestellten politischer Organisationen, besonders der Gruppe der Angestellten der politischen Parteien und Fraktionen. Demzufolge scheint der Bereich der Politik für eine langfristige Karriere der einzelnen Abgeordneten immer bedeutsamer zu werden: Immer mehr MdBs stammen aus politischen oder politiknahen Berufsfeldern. Diese Entwicklungstrends zu einer verstärkten Rekrutierung der Abgeordneten aus politiknahen Berufsfeldern verdeutlichen den Gegensatz zwischen dem Bedarf von Abgeordneten, die mit parlamentarischen und politischen Abläufen vertraut sind, und dem Repräsentationsanspruch gegenüber der deutschen Bevölkerung. Die Auswirkungen, die diese Entwicklungen auf das Verhältnis zwischen Abgeordneten und Bürgern haben, sollten in der Zukunft stärker beobachtet werden. 